\title{
Ensino de física em tempos de pandemia: a utilização do applet "forças e movimento", da plataforma phet interactive simulation, como ferramenta metodológica.
}

\author{
Teaching physics in times of pandemic: the use of the applet "forces and movement", of the phet \\ interactive simulation platform, as a methodological tool.
}

Enseñanza de la física en tiempos de pandemia: el uso del applet "fuerzas y movimiento", de la plataforma de simulación interactiva phet, como herramienta metodológica.

Tárico Barbosa de Freitas

ORCID: https://orcid.org/0000-0001-7201-8101 Universidade Federal dos Vales do Jequitinhonha e Mucuri, Brasil Secretaria de Estado de Educação de Minas Gerais, Brasil E-mail: tarico.freitas@educacaom.g.gov.br

Stênio Cavalier Cabral

ORCID: https://orcid.org/0000-0001-5241-9776

Universidade Federal dos Vales do Jequitinhonha e Mucuri, Brasil

E-mail: stenio.cavalier@hotmail.com

Sérgio Antônio Brum Junior

ORCID: https://orcid.org/0000-0002-4946-7993

Universidade Federal da Integração Latino-Americana, Brasil

E-mail: sergio.junior@unila.edu.br

\begin{abstract}
Resumo
O presente estudo teve por objetivo conhecer e elaborar estratégias didáticas que possibilitem ensinar os conceitos da física de maneira envolvente e promover aprendizagem significativa no contexto dos alunos do $1^{\circ}$ ano do ensino médio, durante o período pandêmico da Covid 19. A metodologia proposta teve como objetivo estimular os estudantes a aprender ativamente, por meio da mediação do professor e uso de simulações computacionais, conceitos da disciplina física. Através destas ferramentas oportuniza aos alunos trabalhar com animações das representações virtuais dos fenômenos. O applet "Forças e Movimento" é relevante para a compreensão de conceitos físicos estudados, pois possibilita que o próprio aluno faça a manipulação desse applet e tire suas próprias conclusões sobre o assunto. Palavras-chave: Ensino; PhET; Força e movimento; Física.
\end{abstract}

\begin{abstract}
This study aimed to understand and develop didactic strategies that enable teaching physics concepts in an engaging way and promote meaningful learning in the context of 1st year high school students during the Covid 19 pandemic period. The proposed methodology will aim at encourage students to actively learn, through the mediation of the teacher and the use of computer simulations, concepts of the physical discipline. Through these tools it will be possible to give students the opportunity to work with animations of virtual representations of phenomena. The "Forces and Motion" applet is relevant for understanding the physical concepts studied, as it allows the student to manipulate this applet and draw their own conclusions about the subject.
\end{abstract}

Keywords: Teaching; PhET; Force and movement; Physics.

\section{Resumen}

Este estudio tuvo como objetivo comprender y desarrollar estrategias didácticas que permitan enseñar conceptos de física de una manera atractiva y promover un aprendizaje significativo en el contexto de los estudiantes de primer año de secundaria durante el período de la pandemia de Covid 19. Animar a los estudiantes a aprender activamente, a través de la mediación del maestro. y el uso de simulaciones por computadora, conceptos de la disciplina física. A través de estas herramientas, brinda a los estudiantes la oportunidad de trabajar con animaciones de representaciones virtuales de fenómenos. El subprograma "Fuerzas y movimiento" es relevante para comprender los conceptos físicos estudiados, ya que permite al alumno manipular este subprograma y sacar sus propias conclusiones sobre el tema.

Palabras clave: Enseñanza; PhET; Fuerza y movimiento; Física. 


\section{Introdução}

A situação de pandemia, originada pelo surgimento e disseminação do novo Coronavírus, o que causa nas pessoas a doença Covid 19, trouxe para a sociedade mundial reflexos e dificuldades que podem ser notados nos mais diversos setores como a prestação de serviços da área de saúde, economia, e no setor educativo.

Com o objetivo de se conter a propagação do agente causador do Covid 19, não só no Brasil, mas no mundo inteiro foram adotados medidas e protocolos de distanciamento social, dentre os impactos causados por todas estas mudanças propostas pelo período pandêmico, destaca-se o surgimento de um novo modelo de ensino, que culminou com a interrupção das aulas na forma presencial em todo o sistema educacional, causando prejuízos especialmente na rede pública de ensino brasileira, através da adoção do ensino remoto, sem que as famílias e escolas estivessem preparadas para este. Acerca do modelo de ensino, Silva \& Peixoto (2020), entendem que o sistema almeja soluções remotas para a educação utilizando como suporte plataformas digitais que sejam disponíveis, confiáveis e proveitosas para este período de crise.

De acordo com Senhoras (2020), a implementação do distanciamento social, como medida de contenção da dispersão do novo Coronavírus, o uso das TDIC aumentou repentinamente em todos os aspectos da vida humana. Nossas relações sociais, profissionais e educacionais que já estavam, antes da pandemia, quase completamente integradas com essas tecnologias, hoje dependem diretamente delas.

No contexto da pandemia, a web desempenha um papel de valorosa importância, uma vez que, sem esse recurso, seria impossível ministrar aulas e/ou compartilhar atividades remotamente. Assim, os novos desafios impõem o uso de desktops, laptops, celulares, tablets ou símiles para que se permita desenvolver o ensino remoto emergencial Reis, Amaro \& Reis (2020).

Segundo Carvalho, Carvalho \& Barbosa (2021) O espaço escolar tem vivenciado fortes mudanças, como a expansão da informação, o avanço das tecnologias e a crescente globalização de mercados. Tudo isso afeta de forma significativa a escola, entretanto, nada foi tão estarrecedor e urgente, quanto à crise sanitária em decorrência da infecção humana pelo novo Corona vírus (COVID-19). Nesse sentido, quando as aulas presenciais foram suspensas os docentes enfrentaram o desafio de reelaborar sua prática pedagógica para os processos formativos se manterem cristalinos Monteiro (2020); Barbosa, Viegas \& Batista (2020); Quadros \& Cordeiro (2020); Machado (2020);

Muitos desafios podem ser encontrados no Ensino de Física durante a pandemia Covid 19, como a falta de interesse por parte dos alunos, a dificuldade em se relacionar os conteúdos e a realidade, dentre muitos outros Moreira (2021); Gonzaga, Miranda \& Ferreira (2020). Infelizmente, a Física ainda é aplicada de maneira desconexa com o cotidiano do aluno, fator que acaba impedindo o aluno de relacionar os conhecimentos aprendidos em sala, e o que acontece no mundo, e que, além disso, não traz um desafio cognitivo que instigue o aluno a aprender os conteúdos científicos Pereira \& Moreira (2017).

O presente estudo trata da análise sobre o applet "Forças e Movimento" disponível em PhET Interactive Simulations. Ele possui diversos exemplos matemáticos e físicos importantes para a contextualização de uma variedade de assuntos em sala de aula, os quais, muitas vezes, são de difícil compreensão por parte do estudante pelo fato de serem, à primeira vista, muito abstratos.

O estudo vai ao encontro das carências apresentadas durante a pandemia causada pelo COVID-19, pois os recursos midiáticos são utilizados por professores e aprendentes, sendo necessários e urgentes no auxílio aos processos de ensino e aprendizagem da disciplina física.

Lopes \& Feitosa (2009) defendem que "[...] o ensino e a aprendizagem de Física podem ser ressignificados pela utilização de recursos tecnológicos". Vogler, Junior \& Germano (2004) complementam, afirmando que "o processo ensinoaprendizagem de Física torna-se muito mais interessante na medida em que simulações são utilizadas".

Sendo assim, empreendeu-se uma investigação qualitativa, sob a forma de uma pesquisa bibliográfica exploratória, que teve o intuito de responder à seguinte questão: O uso do applet "Forças e Movimento", disponível na plataforma PhET Interactive 
Simulation, será capaz de despertar o interesse e promover a aprendizagem significativa, possibilitando uma aprendizagem efetiva em estudantes do ensino médio na disciplina Física durante o ensino remoto? Sendo o objetivo da pesquisa conhecer e elaborar estratégias didáticas que possibilitem ensinar os conceitos da física de maneira envolvente e promover aprendizagem significativa no contexto dos alunos do $1^{\circ}$ ano do ensino médio, por meio de uma metodologia que contemple a utilização do applet como ferramenta metodológica para potencializar o processo educativo durante as aulas de Física no contexto do isolamento social imposto pela pandemia Covid 19.

Desta forma, acreditamos que a interação do estudante com as Simulações Computacionais como ferramenta auxiliar no processo ensino/aprendizagem de Física no modelo de ensino remoto, permita ao estudante trabalhar de forma participativa com os conceitos e relações, tornando-se parte do seu processo de aquisição do conhecimento e, assim, desenvolver uma aprendizagem significativa.

\section{O Ensino de Física através da Experimentação em período pandêmico}

Desde os primeiros passos do Homem na Terra, as experiências por ele feitas passaram a se multiplicar sem qualquer freio, sejam elas para o seu conforto ou sua defesa. Foram criadas ferramentas que proveram comidas, transportes, abrigos, roupas, armas entre tantas outras descobertas que facilitaram o seu dia a dia Cruz (2009). Dissertando ainda sobre esse assunto, essa autora expõe que:

Cada vez mais o homem se diferenciava dos outros animais. A descoberta do fogo mudou não apenas o modo de vida dos nossos antepassados, mas também sua forma de pensar. Ele precisava criar, imaginar, ir em busca de soluções para os afazeres diários; necessitava, e continua necessitando, de oportunidade e tempo para se desenvolver integralmente. É notório, portanto, que as conquistas realizadas contribuíram para a evolução do conhecimento humano e se estendem até a atualidade, ampliando as possibilidades de conquistas futuras. IBID, (p.10-11).

A prática de experiências no decorrer do ensino da Física é de suma importância, mas para que tal prática tenha êxito é preciso ter ciência da validade das experiências a serem executadas. Muitas das vezes, uma experiência procura ilustrar um determinado fenômeno exposto pelo professor, em outro momento aponta para as dificuldades de compreensão de uma determinada teoria. É importante perceber, também, que a prática de experiências requer um engajamento de muitos: professores, alunos, coordenadores e escola, pois, com tal comprometimento, proporcionarão um crescimento intelectual de todos os envolvidos.

Pensando assim, se faz necessário no ensino da Física ter experimentos que venham corroborar no entendimento dos fenômenos apresentados e/ou investigados. Logo, o professor é a figura principal para cumprir a tarefa de instruir os alunos nos procedimentos experimentais para que se faça as inter-relações entre teoria e prática Laburú, Salvadego \& Barros (2009).

\section{As TIDC'S e o Ensino de Física}

A Base Nacional Comum Curricular (BNCC) traz que o Ensino Médio deve fazer uso amplo das TICs para promover a compreensão e a apropriação pelos educandos do modo que as Ciências da Natureza se expressam, na qual se encaixa a Física, garantindo seu envolvimento nos processos de comunicação e divulgação do conhecimento científico (BRASIL, 2018).

As Tecnologias Digitais de Informação e Comunicação (TDIC), em seu histórico de existência, nunca foram utilizadas com tanta veemência para fins educacionais como atualmente. A rede de educação particular e pública, buscam se adequar em todos os níveis a este repentino modo de ensino, que o distanciamento social proporcionou. 
Vivemos atualmente em uma sociedade guiada por avanços tecnológicos, onde o computador tem sido ferramenta de principal utilização. De acordo com Schuhmacher et al (2002), a evolução dos computadores nas últimas décadas, trouxeram mudanças significativas em diversas áreas, mostrando a imensa capacidade que eles oferecem. Os computadores se encontram inseridos no desenvolvimento da Física nos últimos anos, independentemente da sua natureza teórica ou experimental, sendo claramente perceptível a sua imensa influência na resolução de grandes problemas.

Este desenvolvimento tecnológico, aliado ao isolamento social imposto pela situação pandêmica enfrentada atualmente, tem modificado profundamente o cotidiano das pessoas, e a escola não poderia ficar alheia a essa realidade, foi preciso se adaptar e ensinar ao aluno como conviver com essas novas tecnologias (TDIC'S) também no contexto escolar, e se adaptar ao novo modelo educacional proposto durante o período.

Dentro do contexto de isolamento social, o uso de tecnologia se apresentou como uma alternativa, tanto para os alunos quanto para os professores, não somente no sentido de facilitar o trabalho docente, mas garantir maior acessibilidade no desenvolvimento da cultura, da pesquisa e permitir uma maior interação e disciplina no ato de estudar. Com isso observa-se que o desenvolvimento de aulas práticas mediada por aparatos digitais não são apenas importantes, mas sim uma necessidade para acompanhar os avanços tecnológicos da nossa sociedade Sousa, Moita \& Carvalho (2011).

Evangelista \& Gonçalves (2013) afirmam que, mediante esses aparatos, é viável ministrar assuntos diversos, de áreas afins, onde os estudantes podem desenvolver aprendizagem, motivados pela busca do conhecimento, certamente promovendo uma maior interação, neste período de pandemia, com o conteúdo programático.

Segundo Santana \& Sales (2020) no âmbito da educação práticas pedagógicas emergentes surgem para lidarmos com a pandemia. Essas práticas acabam por trazer maiores desafios, além daqueles que o segmento já vinha enfrentando. No ensino de física, com a necessidade de soluções imediatas para o desenvolvimento das aulas práticas em tempos de pandemia, novas estratégias, procedimentos e técnicas foram ocupando espaço nas rotinas das escolas. Esta paralisação imediata trouxe ao centro do debate educacional, o uso das tecnologias educacionais para realização de atividades escolares não presenciais Vieira \& Ricci (2020).

Nesse contexto, torna-se fundamental o uso de tais recursos no ensino de Física, objetivando a transmissão, promoção, interação e socialização do conteúdo a ser apresentado, inspirando os discentes no processo de formação de conceitos da referida disciplina. $\mathrm{O}$ docente assume neste processo a significativa incumbência de mediar através de mecanismos que potencialmente irão privilegiar um ensino e aprendizagem efetivo, que permite acesso sem restrições de localização e horário

\section{A Utilização do PhET como Objeto de Aprendizagem de Física}

O PhET Interactive Simulations é um laboratório virtual que possui inúmeras simulações de experimentos científicos. O software foi desenvolvido por uma Universidade do Colorado em Boulder (University of Colorado at Boulder) localizada nos Estados Unidos da América.

São simulações divertidas e interativas, de fenômenos físicos que servem para aperfeiçoar o entendimento dos conteúdos ministrados de uma forma prática facilitando assim o aprendizado do aluno e absorção dos conteúdos Santos, Alves \& Moret (2006).

O software é disponibilizado gratuitamente em seu portal (https://phet.colorado.edu), sendo disponibilizado também em português pelo sítio (https://phet.colorado.edu/pt_BR/). As experiências nele disponível têm embasamento teórico, sendo revisado periodicamente para tornar sua fidelidade à teoria trabalhada. Dentro deste contexto, Soares (2013) comenta que: "Para garantir a eficiência educacional e usabilidade do software, todas as simulações são exaustivamente testadas e avaliadas".

Ainda sobre esta temática, Arantes, Miranda \& Studart (2010) afirmam que: 
O grupo PhET possui uma abordagem baseada em pesquisa, na qual as simulações são planejadas, desenvolvidas e avaliadas antes de serem publicadas no sítio. As entrevistas com estudantes são fundamentais para o entendimento de como eles interagem com simulações e o que as torna efetivas educacionalmente. Arantes, Miranda \& Studart (2010).

As simulações virtuais no processo se ensino e aprendizagem, assumem um papel preponderante nas aulas facilitando a apreensão de conteúdos facilmente, assim como pode favorecer a interação da turma, o espírito de cooperatividade e competitividade Freitas \& Mancini (2019)

O uso dos simuladores PhET são de grande utilidade. Conforme Carraro \& Pereira (2014):

O uso dos simuladores virtuais do PhET como recursos didáticos no ensino de Física pode contribuir significativamente para a aprendizagem dos conteúdos físicos, pois age como facilitador e motivador no processo de ensino e aprendizagem. Busca-se colocar o estudante mais ativo no processo de ensino de forma que observe os modelos físicos, avance na construção de conceitos, leis e teorias, colete dados das simulações, elabore hipóteses e teste a validade das mesmas, confronte o seu conhecimento prévio com o conhecimento científico, questione, estabeleça relação entre a teoria e prática na compreensão dos fenômenos físicos presentes no seu dia a dia.

Portanto, o uso do software pode ser de grande auxílio para o professor da educação básica, que necessita da utilização de experimentos para fundamentar e aperfeiçoar sua metodologia de ensino, procurando atribuir significado ao conceito físico, inter-relacionando-o com o cotidiano do estudante, dando significado a teoria que está sendo trabalhada, através de recursos que possibilitem o aluno observar que tudo o que ele está aprendendo está visivelmente no seu contexto social.

\section{Material e Métodos}

A pesquisa é um processo permanente e faz possível a aproximação do investigador com a realidade a investigar. Esta aproximação fornece subsídios para uma intervenção realizada com o objetivo de resolver um problema ou indicar caminhos Gerhardt \& Silveira (2009).

A proposta aqui apresentada, trata-se de uma metodologia de ensino que auxilie professores e alunos no processo aprendizagem no modelo de ensino proposto durante o atual período de isolamento social, através de uma sequência didática de atividades baseadas em uma situação real e contextualizada na realidade do aluno. Neste sentido os alunos são confrontados com situações problemas que tem o intuito de desafiá-los e principalmente provocar sua curiosidade.

A escolha do tema baseou-se no desejo de possibilitar ao aluno mesmo estando vivendo em meio a pandemia Covid19, explorar situações do seu dia a dia, por meio de um enfoque experimental, no qual buscou-se oportunizar a construção da aprendizagem de conceitos físicos baseados na Mecânica Newtoniana. Após a escolha do tema, foi definida a simulação, optando pelo uso da "Força e Movimento: Noções Básicas." disponível em http://phet.colorado.edu/pt_BR/simulation/forces-andmotion-basics. Ela se encontra dividida em quatro partes, o que chamaremos de ambientes e sua seleção é feita através de abas presentes na parte superior da tela inicial como podemos perceber na (Figura 1), neste estudo adotaremos como objeto o ambiente "cabo de guerra". 
Figura 1 - Tela Inicial da simulação Força e Movimento: Noções Básicas.

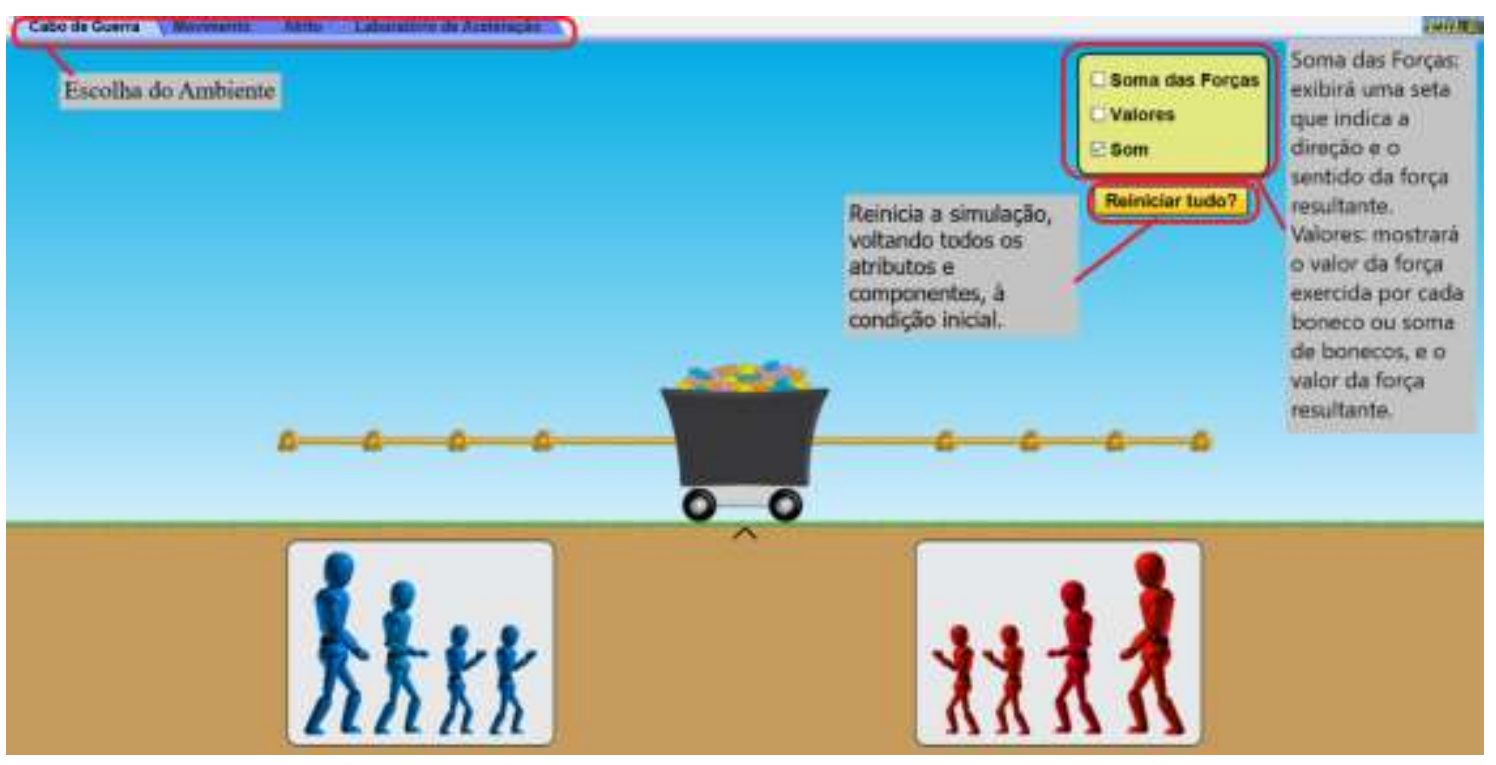

Fonte: Autores (2021).

Vale ressaltar que, estas simulações apresentam uma contextualização natural para os estudantes, o que ao nosso ver facilita como ponto de partida na construção do conhecimento e a exploração do conhecimento entre o que os alunos pensam e o modelo científico, que é o que sabemos sobre este tema.

\section{Ambiente 1 - Cabo de Guerra}

Nesta opção encontraremos conforme mostrado na (Figura 2), na área principal um carrinho e duas equipes com bonecos vermelhos, a direita e azuis, a esquerda, o que nos permitirá investigar a diferença entre forças em equilíbrio e desequilíbrio, à medida que os puxadores competem em um cabo de guerra por um carrinho de doces., O tamanho dos bonecos disponíveis na simulação é proporcional à quantidade de força que eles irão aplicar (pequeno $=50 \mathrm{~N}$, médio $=100 \mathrm{~N}$, grande $=150 \mathrm{~N}$ ) e o jogo do cabo de guerra termina quando o carrinho bate a rodinha no batente.

Ao darmos início a simulação, arrastarmos qualquer boneco para a corda posicionando os dos dois lados e clicamos em “Iniciar" e assim será iniciada disputa do cabo de guerra entre os bonecos, a simulação se apresenta de forma bem semelhante a tradicional brincadeira, após acionado o botão iniciar é substituído pelo botão "Parar" que interrompe a disputa e também ficará disponível o botão "Voltar" que retornará a simulação exatamente ao momento antes de as equipes começarem a exercer força. O uso desta simulação potencializará ao estudante o entendimento sobre os conceitos da mecânica newtoniana, permitindo-o a fazer inferências como:

- $\quad$ Observar que a força aplicada sobre um objeto pode fazer com que ele se mova;

- Concluir que a força resultante é o resultado da soma das forças atuantes;

- $\quad$ Interpretar o movimento de um objeto com força resultante zero. 
Figura 2 - Tela de execução da simulação cabo de guerra.

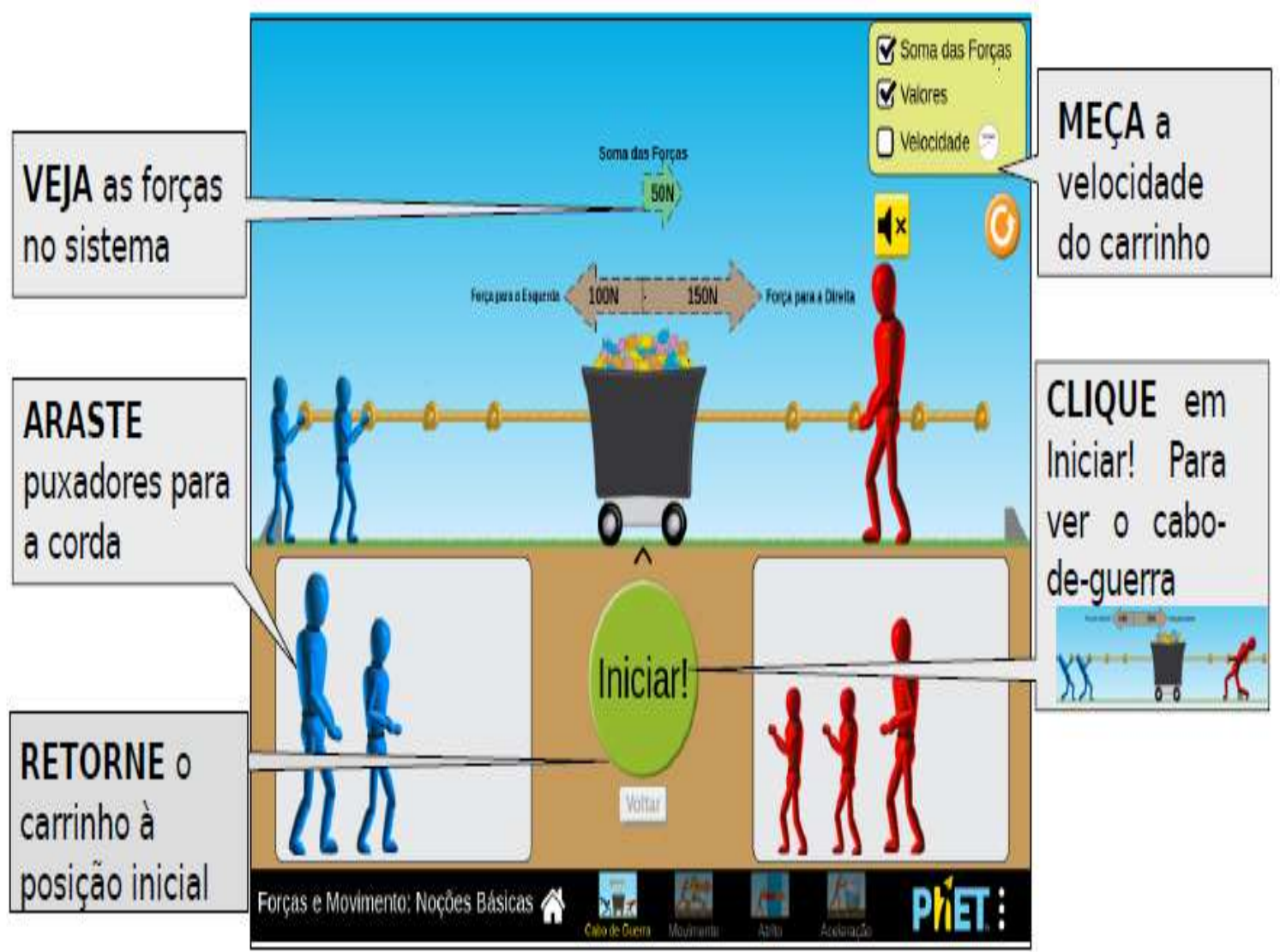

Fonte: Autores (2021).

\section{Considerações Finais}

Com o intuito de auxiliar a aprendizagem da Física no $1^{\circ}$ ano do Ensino Médio, especificamente sobre os conteúdos da mecânica newtoniana, este estudo buscou promover um embasamento teórico à utilização das simulações e experimentação como ferramenta de ensino de Física, visando proporcionar condições que unissem teoria e prática na construção de uma aprendizagem mais significativa no ensino remoto proposto pelo isolamento social causado pela Covid 19.

Conforme citado em diversas partes deste trabalho, o uso das tecnologias midiáticas em aulas de Física, pode facilitar a aprendizagem do aprendiz, uma vez que ela traz, de forma prática, clara e dinâmica, todos os objetos de conhecimento estudados nesta disciplina. Além disso, neste momento de pandemia se faz necessário lançar mão de ferramentas que extrapolem o uso apenas de quadro e giz, metodologia indisponível enquanto perdurar o isolamento social, imposto pela crise sanitária do Covid 19.

O aluno traz consigo assuntos que já são conhecidos, e é no momento das aulas que assimila esses conceitos e que tudo passa a ter significado. Uma vez que o professor traz os temas teóricos para os alunos e depois apresenta uma plataforma como o PhET, onde todos os objetos de conhecimento estudados teoricamente farão sentido para o estudante e ele compreenderá a sua aplicação.

Especificamente, o applet "Forças e Movimento", que foi objeto de estudo e análise até aqui, traz essa dinâmica, interação e tecnologia em um único espaço, podendo tornar a aula ainda mais atraente. 
Esse applet possui diversos conceitos físicos que auxiliam o estudante na compreensão e pode permitir-lhe manipular os objetos, selecionar os objetos de conhecimento que deseja visualizar e fazer suas próprias conclusões sobre estes assuntos. Sendo assim, diante das análises realizadas, pode-se responder ao problema de pesquisa apresentado na introdução.

Por fim, este trabalho denota que o professor pode dispor-se a utilizar essa tecnologia midiática durante e após a pandemia como apoiadora e auxiliadora do aprendizado, e que o estudante a compreenda para que essa possa contribuir com o seu desenvolvimento e sua aprendizagem. Nesse sentido, pretende-se investigar futuramente quais recursos digitais os docentes continuaram utilizando em suas aulas pós pandemia, a fim de identificar como eles influenciaram e mudaram o ensino no âmbito do o uso da tecnologia em geral e, de forma mais especifica, no ensino de Física.

\section{Referencias}

Arantes, A. R., Miranda, M. S. \& Studart, N (2010). Objetos de Aprendizagem no ensino de Física: usando simulações do PheT. Física na Escola, v. 11, n. 1,. http://www.sbfisica.org.br/fne/Vol11/Num1/a08.pdf.

Barbosa, A. M., Viegas, M. A. S. \& Batista, R. L. N. F. F. (2020). Aulas presenciais em tempos de pandemia: relatos de experiências de professores do ensino superior sobre as aulas remotas. Revista Augustus. 25(51). https://revistas.unisuam.edu.br/index.php/revistaaugustus/article/view/565

BRASIL (2018). Ministério da Educação. Secretaria da Educação Básica. Base Nacional Comum Curricular. Brasília, DF. http://basenacionalcomum.mec.gov.br/images/BNCC_EI_EF_110518_versaofinal_site.pdf

Carraro, F. L., \& Pereira, R. F. (2014). O uso de simuladores virtuais do PhET como metodologia de ensino de eletrodinâmica. In: Os desafios da escola pública paranaense na perspectiva do professor PDE, $\quad$ Cadernos http://www.diaadiaeducacao.pr.gov.br/portals/cadernospde/pdebusca/producoes_pde/2014/2014_uem_fis_artigo_francisco_luiz_carraro.pdf.

Carvalho, E.P.N. de., Carvalho, P.L. de, \& Barbosa, L.L.C. (2021). A relevância e os cenários da supervisão pedagógica frente ao ensino híbrido no contexto pandêmico do COVID-19 no Tocantins. Research, Society and Development, 10 (12), e588101221055. https://doi.org/10.33448/rsd-v10i12.21055

Cruz, J. B. da. (2009). Laboratórios. Brasília: Universidade de Brasília

Evangelista, T. B. N \& Gonçalves, P. R. (2013). O uso da tecnologia como uma ferramenta para despertar o interesse por aprender: um estudo de caso prático. 09. ed. Rio Claro - São Paulo: Revista Tecnologias Na Educação.

Freitas, J. L. A., \& Mancini, K. C. (2019). Contribuições da ludicidade no processo de ensino e aprendizagem de conhecimentos científicos e culturais.KiriKerê: Pesquisa em Ensino, 7, 104-122.

Gerhardt, T.E. \& Silveira, D.T. (2009). Métodos de pesquisa / [organizado por Tatiana Engel Gerhardt e Denise Tolfo Silveira]; coordenado pela Universidade Aberta do Brasil - UAB/UFRGS e pelo Curso de Graduação Tecnológica - Planejamento e Gestão para o Desenvolvimento Rural da SEAD/UFRGS. - Porto Alegre: Editora da UFRGS.

Gonzaga, G. R., Miranda,J. C. \& Ferreira,M. L. (2020). Teaching the theme periodic table in basic education. Research, Society and Development,9 (1), e97911657

Lopes, R. \& Feitosa, E. (2009). Applets como recurso pedagógico no ensino de física. Aplicação em cinemática. http://www.cienciamao.usp.br/dados/snef/appletscomorecursopedago.trabalho.pdf.

Machado, D. P. (2020). Educação em tempos de covid - 19: reflexões e narrativas de pais e professores. Editora Dialética e Realidade, Curitiba. https://2b0ee3ca-fda1-4c02-aa8d-d6226f4481c2.filesusr.com/ugd/206e81_0a66ffe51a3a49eea20692bb92d96b64.pdf

Monteiro, S. S. (2020). (Re)inventar educação escolar no brasil em tempos da COVID-19. Rev. Augustus 25 (51).

Moreira, M. A. (2021). Desafios no ensino da física. Revista Brasileira de Ensino de Física, 43

Pereira, M. V. \& Moreira, M. C. A. (2017). Atividades prático-experimentais no Ensino de Física. Caderno Brasileiro de Ensino de Física. $34(1), 265-277$.

Quadros, D., Cordeiro, G. R. (2020). Pais, filhos e escola Ressignificação em tempos de pandemia. https://www.aredacao.com.br/artigos/133588/a-escoladosfilhos- em-tempos-de-pandemia.

Reis, A.V.S.A, Amaro, D. \& Reis, F. (2020). Ensino remoto: o planejamento das aulas é, mais do que nunca, uma necessidade. https://revistaensinosuperior.com.br/planejamento-das-aulas/

Santana, C. L. S. E, \& Sales, K. M. B. (2020). Aula em casa: educação, tecnologias digitais e pandemia covid-19. Interfaces Científicas - Educação, 10 (1), 75 92. https://doi.org/10.17564/2316-3828.2020v10n1p75-92

Santos, G. H., Alves, L. \& Moret, M. A. (2006). Modellus: Animação Interativas mediando a Aprendizagem Significativa dos Conceitos de Física no Ensino Médio. Revista Científica da escola de administração do exército, v. 2, p. 88-108.

Schuhmacher, et al. (2002). Experiências Virtuais Aplicadas em Aulas de Teoria de Física. http://inf.unisul.br/ ines/workcomp/cd/pdfs/2810.pdf>. 
Research, Society and Development, v. 10, n. 15, e220101522796, 2021

(CC BY 4.0) | ISSN 2525-3409 | DOI: http://dx.doi.org/10.33448/rsd-v10i15.22796

Senhoras, E. (2020). Coronavírus e Educação: Análise dos Impactos Assimétricos. - Boletim de Conjuntura, Ano II | Volume 2 | No 5 | Boa Vista.

Silva, F. C. S. \& Peixoto, G. T. B. (2020). Percepção dos professores da rede estadual do Município de São João da Barra-RJ sobre o uso do Google Classroom no ensino remoto emergencial. Research, Society and Development, v. 9, n. 10, p. 1-24.

Sousa, R. P. de, Moita, F.M.C. da S. C.; Carvalho, A. B. G. (2011). Tecnologias Digitais Na Educação. 21. ed. Campina Grande-PB: Editora da Universidade Estadual da Paraíba-EDUEPB. 278 p.

Vieira, L.,Ricci, M.C. C. (2020). A educação em tempos de pandemia: soluções emergenciais pelo mundo. OEMESC -Observatório do ensino médio em Santa Catarina. Editorial de abril. https://www.udesc.br/arquivos/udesc/id_cpmenu/7432/EDITORIAL_DE_ABRIL__Letícia_Vieira_e_Maike_Ricci_final_15882101662453_7432.pdf.

Vogler, M., Junior, F. S. \& Germano, J. S. E. (2004). O uso de simulações em Java como objetos de aprendizagem no ensino de física. http://www.abenge.org.br/cobenge/arquivos/15/artigos/01_504.pdf. 